\title{
Progress toward Operando TEM using EELS
}

\author{
B.K. Miller and P.A. Crozier \\ School for Engineering of Matter, Transport and Energy, Arizona State University, Tempe, AZ \\ 85287-6106
}

Operando techniques combine the simultaneous characterization of a catalyst and its activity. Insitu transmission electron microscopy (TEM) is a powerful technique for observing catalysts at the atomic level. However, until recently, quantitative measurement of catalytic activity during this characterization had not been attempted. We are developing a technique that uses electron energy loss spectroscopy (EELS) to analyze the gas composition in an environmental TEM, thus allowing reactions to be monitored in real time.

While the initial results published by Chenna and Crozier [1] on EELS of catalytically produced gases are promising, the initial experiments performed inside the environmental TEM (ETEM) cell have brought to light some difficulties with the use of a differentially pumped system. Small apertures are located in the TEM column to maintain high pressure at the sample, while keeping gas from reaching the electron gun. Different gas species flow through these apertures at different rates, according to their molecular mass, leading to a partial pressure ratio inside the ETEM cell which is different from the nominal composition. In general, each gas flows differently through the entire system, and so these effects must be taken into account when introducing precise gas mixtures into the cell or attempting to analyze those currently inside [2].

Despite these issues, accurate composition determination at the sample should still be achievable. Since the energy loss spectrum is generated largely from a small volume around the sample, the composition measured by EELS should be quite close to that which the sample experiences, even if this is not the composition which was input into the cell. This is in stark contrast to another more sensitive technique we are pursuing: operando TEM by mass spectrometry, in which the gas is measured far from the sample, and may vary significantly in composition from the gas around the sample, leading to further challenges.

While semiquantitative gas compositions can now be routinely determined in our microscope, and these compositions are measured from a small volume around the sample, achieving precise quantitative results necessitates careful testing of the system to characterize its response. As an example, the data from one of these tests is shown in Figures 1 and 2. A nominally one to one mixture of $\mathrm{H}_{2}$ and $\mathrm{O}_{2}$ is flowed into the cell over a period of $90 \mathrm{~min}$. After correcting for spectral drift and background intensity variation in the EELS signal, a linear combination based fit is found, and yields a plot of composition over time, showing clearly the non-ideality of the system. We will discuss the results of this and other tests which are being performed to fully elucidate the difficulties with precise quantification.

References:

[1] S. Chenna \& P.A. Crozier, ACS Catalysis 2, (2012) p.2395-2402.

[2] P.A. Crozier \& S. Chenna, Ultramicroscopy 111, (2011) p.177-185. 
[3] The support from National Science Foundation CBET-1134464 and the use of ETEM at John M. Cowley Center for HR Microscopy at Arizona State University is gratefully acknowledged.
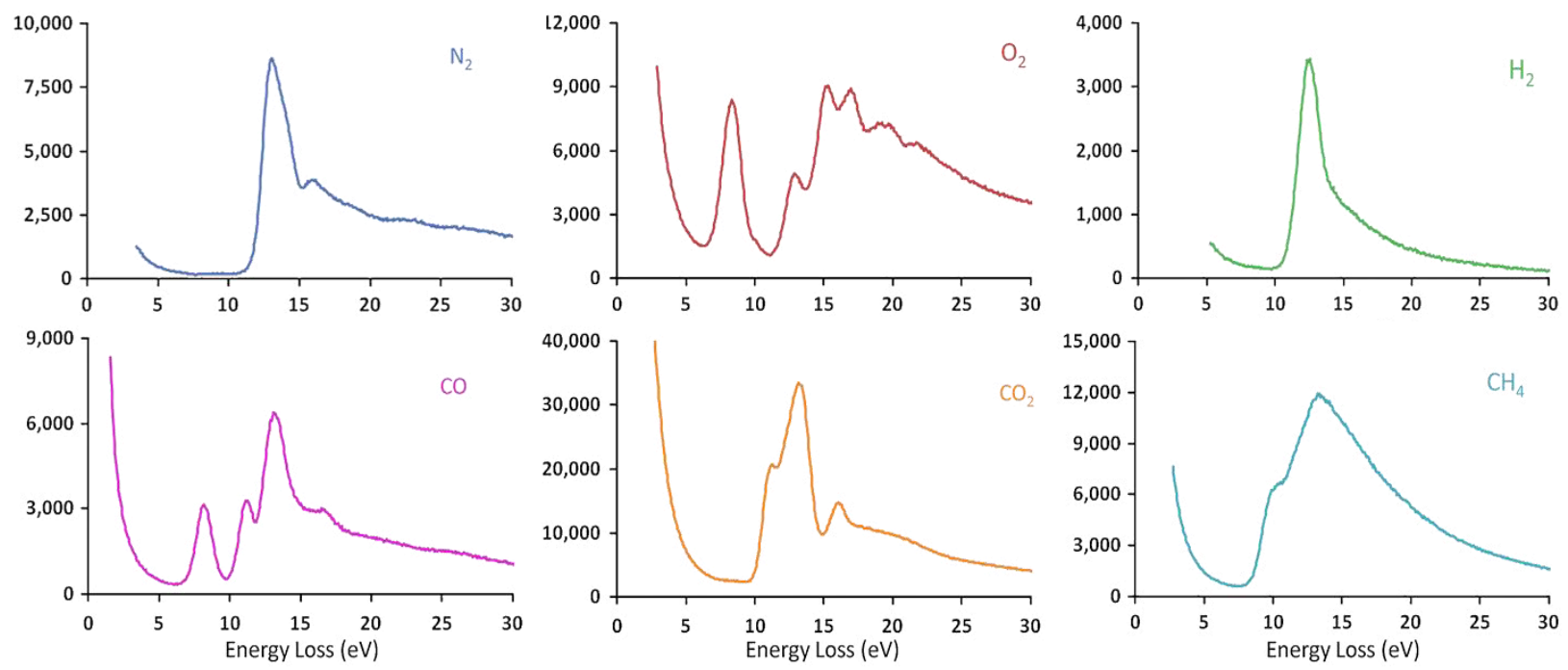

Figure 1. Low loss EELS spectra of various simple gases. Linear combinations of these can be fitted to an experimental spectrum to determine the gas composition present in the ETEM cell.

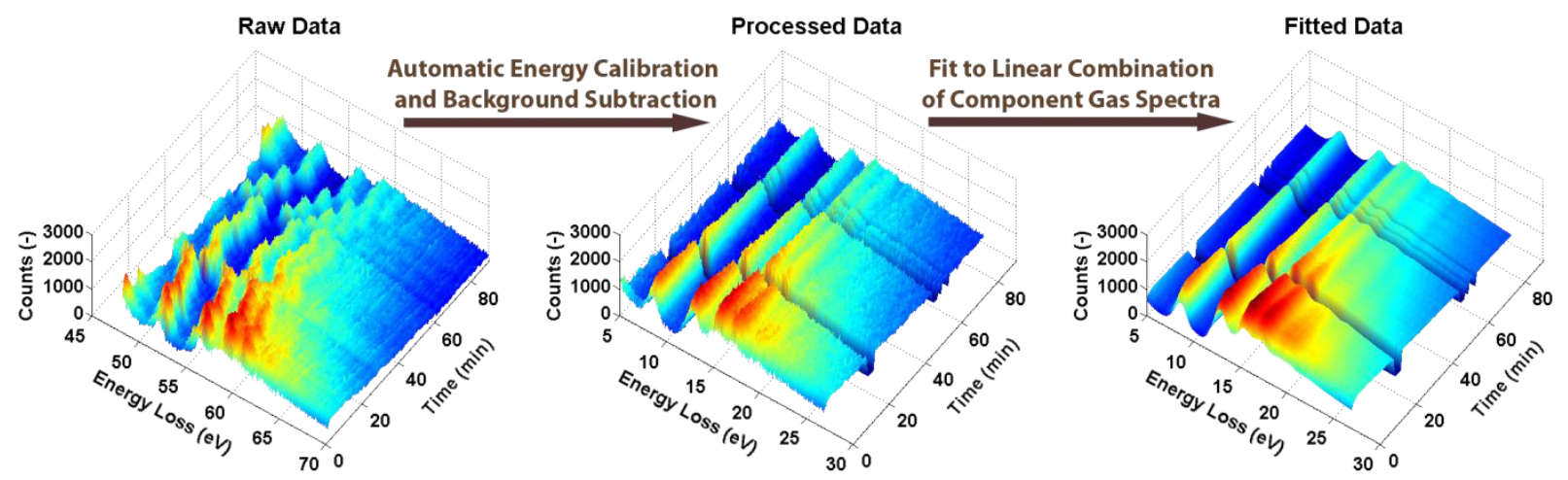

Figure 2. Experimental data obtained from a test mixture of $\mathrm{O}_{2}$ and $\mathrm{H}_{2}$. The raw data is analyzed automatically using MATLAB, which corrects for energy drift in the acquired spectra, and subtracts the background. The data are then fit using a linear combination of the individual gas component spectra shown in Figure 1. The fits are shown in the plot at the right, and the composition is plotted in Figure 3.

Figure 3. The composition calculated from the linear combination fit as a function of time, showing that the $\mathrm{H}_{2}$ content in the cell decreased from its intended $50 \%$ to around $30 \%$ within 20 minutes, due to differential effusion and pumping of different gas species. This and other tests of the system are necessary before precise quantitative analyses of the data from catalytic systems can be performed.

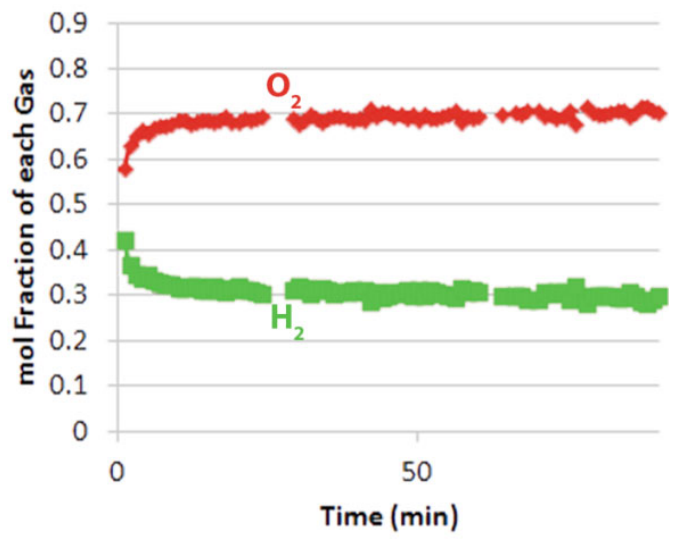

